\title{
3D structure determination from HRTEM and electron diffraction tomography
}

\author{
Xiaodong Zou,*** Daliang Zhang,*** Peter Oleynikov, ${ }^{*}$ Junliang Sun, ${ }^{* * *}$ and Sven Hovmöller* \\ * Structural Chemistry, Stockholm University, SE-106 91 Stockholm, Sweden \\ ** Berzelii Centre EXSELENT on Porous Materials, Stockholm University, SE-106 91 Stockholm, \\ Sweden
}

Electron crystallography is an important complement to X-ray crystallography. There are two main advantages of structure determinations by electron crystallography compared to X-ray diffraction: 1) crystals millions times smaller than what is needed for X-ray diffraction can be studied and 2) the phases of the crystallographic structure factors, which are lost in X-ray diffraction, are present in high resolution transmission electron microscopy (HRTEM) images. Recent developments in instrumentation and methods have tackled the two main weak sides of electron crystallography; the difficulties to obtain complete 3D data and the low quality of diffraction intensities, due to multiple diffraction. Today, the 3D atomic structures of crystals (inorganic, organic or biological) can be accurately determined by electron diffraction and HRTEM combined with crystallographic image processing.

Many zeolites and other complex structures have remained unsolved due to the structure complexity and small crystal sizes. Atoms may overlap in any projection for such structures. In order to resolve atoms, it is necessary to perform 3D reconstruction by combining HRTEM images from several difference projections. We will demonstrate several examples of 3D structure determination from HRTEM images, for zeolite catalysts and mesoporous materials [1-3] (Figs. 1-2). The method is general and can be applied to any crystalline materials, where the crystals are too small or the structures too complicated to be solved by XRPD alone, especially for those crystals containing defects.

We will demonstrate the power of 3D electron diffraction tomography for structure determination (Fig. 3). With this technique, complete 3D electron diffraction data can be collected in a fast and automatic way. Dynamic effects can be significantly reduced when electron beam is tilted away from the crystallographic axes. We are developing software for automatic peak search, unit cell determination and electron diffraction intensity extraction. The data obtained from the 3D electron diffraction tomography can be used for determination of 3D unit cells, the crystal symmetry as well as the structures of unknown crystals.

\section{References}

[1] A. Corma, M. Moliner, Á. Cantín, M. J. Díaz-Cabañas, J.L. Jordá, D.L. Zhang, J.L. Sun, K. Jansson, S. Hovmöller, X.D. Zou, Chem. Mater. 20 (2008) 3218.

[2] Ch. Baerlocher, F.Gramm, L. Massüger, L.B. McCusker, Z.B. He, S. Hovmöller, X.D. Zou, Science 315 (2007) 1113.

[3] Y. Han, D.L. Zhang, L.L. Chng, J.L. Sun, L. Zhao, X.D. Zou, Jackie Y. Ying, Nature Chem. (2009) accepted.

[4] CRISP and ELD, http://www.calidris-em.com/.

[5] Acknowledgement: This work was supported by the Swedish Research Council (VR) and the Swedish Governmental Agency for Innovation Systems (VINNOVA). 


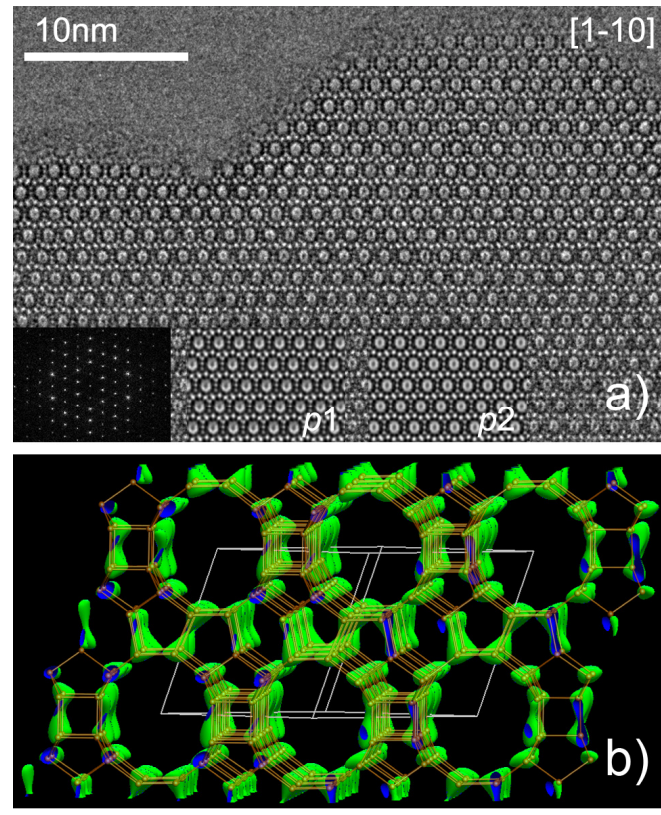

FIG 1. Structure determination of the zeolite beta $(C 2 / c, a=17.90 \AA, b=$ $17.92 \AA, c=14.33 \AA, \beta=114.8^{\circ}$ ) from HRTEM images. a) An HRTEM image taken along the [1-10] direction. The structure factor amplitudes and phases were extracted from the Fourier transform of the image. Insets are Fourier transform, and averaged images with $p 1$ and $p 2$ symmetry imposed, respectively. b) A 3D potential map was reconstructed from those reflections by inverse Fourier transform. All 9 unique Si atoms were obtained from this 3D map.
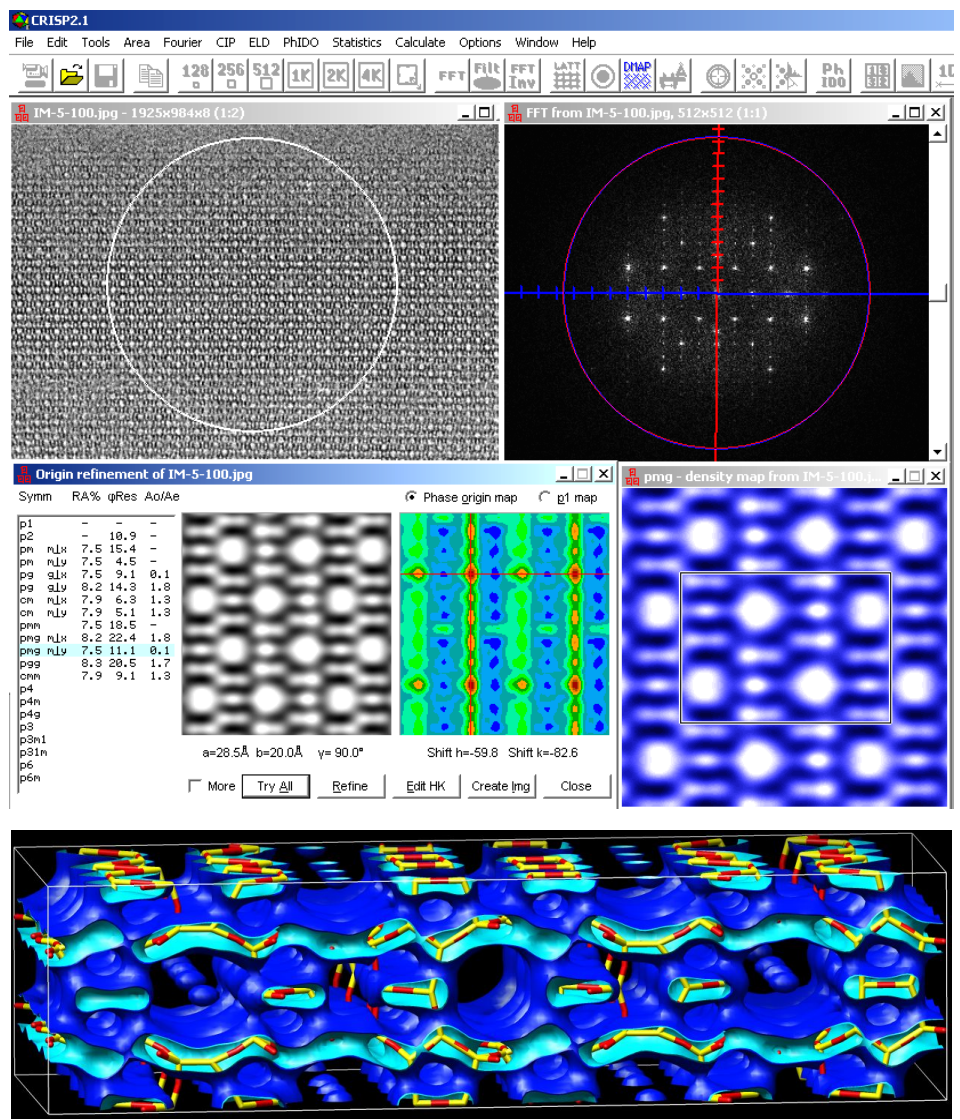

FIG 2. Structure determination of the zeolite catalyst IM-5 (Cmcm, $a=14.3 \AA, b=57.4 \AA, c=20.1 \AA$ ) from $3 \mathrm{D}$ reconstruction. Determination of projection symmetry and retrieval of the structure projection from an HRTEM image taken along the [100] direction by crystallographic image processing using CRISP [4]. 3D potential map reconstructed from 144 reflections using the program eMap. All $24 \mathrm{Si}$ atoms were obtained from this $3 \mathrm{D}$ map. The framework is superimposed.
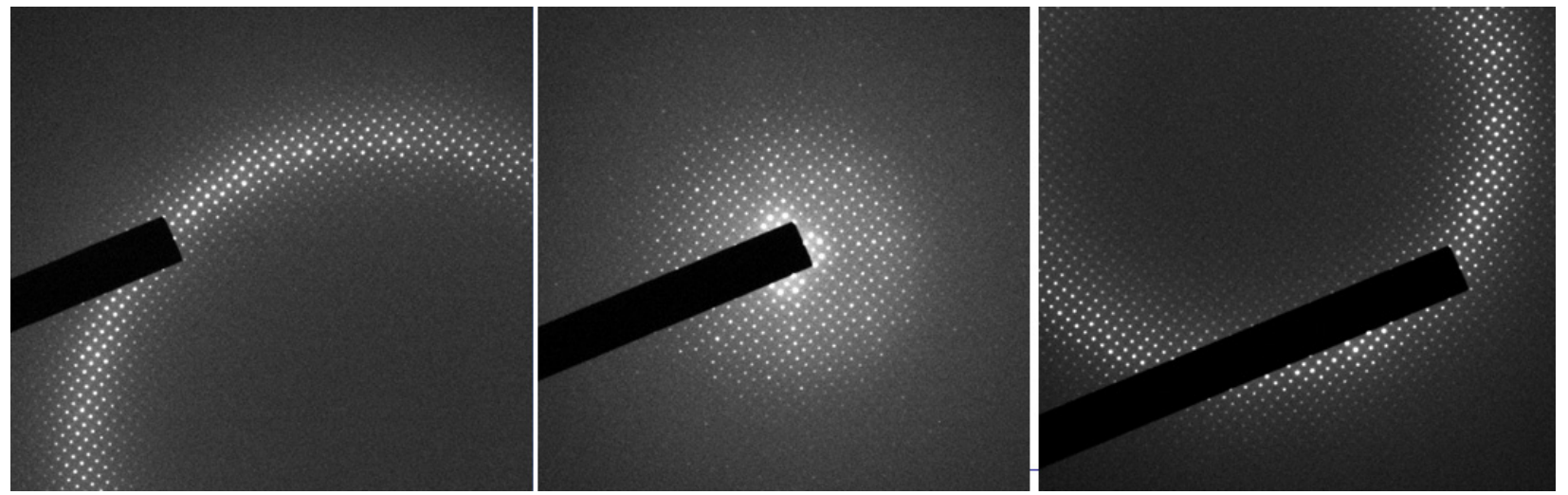

FIG 3. Selected electron diffraction patterns of $\mathrm{K}_{2} \mathrm{O} \cdot 7 \mathrm{Nb}_{2} \mathrm{O}_{5}(P 4 / m b m, a=27.5 \AA, c=3.94 \AA)$ from a tilt series obtained by $3 \mathrm{D}$ electron diffraction tomography. 